\title{
Drug Interaction in Psycho-Oncology: Antidepressants and Antineoplastics
}

\author{
C. Miguel $^{\mathrm{a}} \quad$ E. Albuquerque ${ }^{\mathrm{b}}$ \\ a Psychiatry Service of Hospitals of the University of Coimbra, and ${ }^{b}$ Psychiatry Unit of the Portuguese Institute of \\ Oncology of Coimbra, Coimbra, Portugal
}

\section{Key Words}

Antidepressants $\cdot$ Antineoplastic drugs $\cdot$ Drug interactions

\begin{abstract}
Background and Objectives: Although there is a growing impact of psychiatric and depressive disorders in cancer patients, literature on the idiosyncrasies of antidepressants (ADs) used in those conditions and their interactions with antineoplastic agents (ANs) is scarce. Sharing the same biotransformation pathways enhances the risk of drug interaction between $A D s$ and $A N s$, specifically when compounds are inducers, inhibitors or substrates of cytochrome P450 (CYP 450). In cancer patients, such drug interactions may result in less efficacy of the drug and/or increase of their side effects. Therefore, the choice of AD should be cautious (safe and effective) and well supported. The main purpose of this review was to analyze the individual pharmacokinetic properties of the most used ADs and ANs in order to summarize the risk of possible drug interactions between them, anticipating the consequences of their coadministration. Methods: The authors reviewed books and PubMed online articles published in the last 6 years. Results: Most of the ANs are subject to transformation by CYP $4503 \mathrm{~A} 4$ and their coadministration with $A D s$, that have inhibitory properties of
\end{abstract}

this CYP isoform, such as fluoxetine, sertraline, paroxetine and fluvoxamine, may result in the loss of the AN's efficacy or higher toxicity. Conclusion: Among the ADs, escitalopram, citalopram, venlafaxine, mirtazapine and milnacipran stand out for their weak CYP 450 inhibitory potential and their safety profile in those patients.

Copyright $\odot 2011$ S. Karger AG, Basel

\section{Introduction}

Psychiatric disorders affect about half of the cancer patients [1]. Adjustment disorder is the most frequent diagnosis, followed by major depression, with prevalences going from 4 to $35 \%$ and from 3 to $36 \%$, respectively, according to the diagnostic criteria used, the sample studied (outpatient, pre- or postsurgical), and the type and stage of cancer [2-4].

Several studies have looked at the impact of depression on cancer patients, supporting a significant relationship between depression and lower quality of life, increased perception of pain, suicidal ideas, wish for hastened death, low compliance to treatment and a worse prognosis [5-7]. This evidence was the base of several consensus conferences, guidelines, algorithms and reviews, devel-

\section{KARGER}

Fax +4161306 1234

E-Mail karger@karger.ch

www.karger.com
(C) 2011 S. Karger AG, Basel

0031-7012/12/0886-0333\$38.00/0

Accessible online at:

www.karger.com/pha
Fernanda Cristina Ordens Miguel

Psychiatry Service of Hospitals of the University of Coimbra

Rua Flávio Rodrigues, n ${ }^{\circ} 57,1^{\circ} \mathrm{Dir}^{\text {to }}$

PT-3000-355 Coimbra (Portugal)

Tel. +351 961031 769, E-Mail miguel_fernanda@yahoo.com 
oped with the purpose of promoting and conciliating psychopharmacological and psychosocial treatments, and highlighting the need of evaluating and treating depression in cancer patients $[2,8,9]$.

In fact, the use of antidepressants (ADs) in cancer patients has increased in the last 20-25 years $[2,10]$, and is recommended in the treatment of severe to moderate major depression [11]. Yet, the evidence does not support the supremacy of one AD over the other or a pharmacological treatment over a psychosocial one [11]. Nevertheless, it is widely accepted that the choice of the AD must take into account individual factors, such as side effects, patient's symptoms and preferences, the previous clinical response, and coadministered drugs [11].

In cancer patients particularly, the choice of a psychotropic drug is a challenge that must target 'not to harm'. In fact, those patients are more vulnerable to drug interactions because of their physical fragilities (poor nutrition, weight loss, other medical conditions, young or advanced age), multimedication and possible enzyme deficit due to genetic polymorphisms or iatrogenic causes [12]. Therefore, the choice of psychotropic drugs must be cautious, minimizing the possible increase in adverse side effects and the decrease in the efficacy of drugs which are crucial to the treatment of cancer.

Both ADs and antineoplastic agents (ANs) have narrow therapeutic indices and consequently, small variations in their plasmatic concentrations may result in subtherapeutic or toxic effects [13]. ANs and ADs may interact by pharmacokinetic or pharmacodynamic mechanisms which are independent of the pharmacologic properties responsible for their therapeutic efficacy (i.e. mechanism of action and 'class effect') [14].

Although pharmacokinetic drug interactions between ADs and ANs may result in changes in absorption, distribution or elimination of one of them (when subordinated to the effect of the other), those relevant to clinical practice are attributed mostly to induction or inhibition of cytochrome P450 (CYP 450) occurring in phase I biotransformation [13, 15-17]. In fact, most ANs and ADs are metabolized by CYP 450 enzymes, and it is precisely this sharing of biotransformation pathways that carries a high potential of interaction resulting in an increase or decrease in plasmatic concentrations $[13,15]$.

On the other hand, pharmacodynamic drug interactions occur when the concomitant use of two drugs modifies their pharmacological effect and does not change their pharmacokinetics (plasmatic concentration sustained) [12,13]. Those reactions may be additive, synergic or antagonistic, and are usually predicted ac- cording to the mechanism of action of each agent on the target receptor or enzyme [14]. In fact, in the last decade, in vitro studies have been used to evaluate, understand and anticipate the risk of drug interactions [14]. In spite of the recognized limitations, this emerging knowledge and evidence is of great value in building a descriptive scientific model of the pharmacokinetic and pharmacodynamic behavior of these drugs, allowing to anticipate and to prevent potentially adverse drug interactions [14].

In psycho-oncology, except for the research on the simultaneous use of ADs and tamoxifen (TMX) in breast cancer patients $[18,19]$, the literature is scarce on reviews or research addressing the drug interactions between ANs and ADs and their clinical consequences. Recently, in Singapore, OncoRx has been developed [13]. It is a computer database, still with restricted access, which facilitates the knowledge of probable drug interactions between the main groups of ADs and ANs, used as monotherapy or chemotherapy protocols, based on known pharmacokinetic parameters [13].

Recognizing the importance of this issue, the authors propose to analyze the individual pharmacokinetic profile of the most commonly used ADs and ANs, and pointed out possible interactions between these two groups of drugs, emphasizing the risk of toxicity from antineoplastic activity.

\section{Method}

In this review, papers published after 2005, namely specific books about drug interactions in psychiatry and psychopharmacology and online papers were considered. PubMed articles were retrieved through the association of the following terms: antidepressants, tricyclic antidepressants, depression, anticancer drugs, antineoplastic drugs, tamoxifen and oncology; with the words: metabolism, CYP 450 inhibition and drug interactions. Moreover, for a specific drug (x) whose biotransformation pathway was not clarified by the previous method, an individual research was performed in Google ('x metabolism'). Only two papers aged 12 and 13 years were chosen because of their value to the realization of this review.

\section{Metabolism of Drugs: CYP 450}

Among the 4 major phases which are part of the pharmacokinetic process -absorption, distribution, metabolization and elimination - biotransformation is the one that deserves to be highlighted concerning drug interactions [14]. In this phase, the following possibilities must 
be considered: (1) the 'parent compound' (pro-drug) needs to be metabolized into an active metabolite (ActiveMet) to have a therapeutic effect; (2) the 'parent compound' is active and after metabolization, ActiveMet(s) are formed but with different pharmacologic properties which are usually responsible for side effects; (3) the 'parent compound' is active and deactivated after metabolization [20].

Drug biotransformation depends on 2 phases: phase I (oxidation), which covers redox or hydrolytic reactions responsible for the conversion of lipophilic drugs into more polar metabolites (e.g. alcohols, phenols and carboxylic acids), and phase II reactions of conjugation forming products such as glucuronides and sulfates, readily excreted in urine [14].

Most phase I reactions are catalyzed by CYP 450 localized mostly in the liver. CYPs are a group of about 30 oxidative heterogeneous isoenzymes classified in families by numbers (CYP 1, CYP 2 and CYP 3 ) and in subfamilies by a capital letter, based on the shared amino acid sequences. In humans, the CYP P450 isoforms most involved in the metabolization of drugs are $1 \mathrm{~A} 1,1 \mathrm{~A} 2$, 2A6, 2B6, 2C8, 2C9, 2C19, 2D6, 2E1, 2J2, 3A4, 3A5 and $3 \mathrm{~A} 7$ [14]. The CYP 2D6 and CYP 3A4 isoforms are responsible for metabolizing most psychotropics [12] and they can be subject to induction or inhibition by other drugs or substances.

The evidence shows that the activity of a pro-drug, which is transformed into an ActiveMet, is increased in the presence of an inducer, being the contrary true for those being inactivated after metabolization. However, when a drug interaction changes the concentration of a 'parent drug' and its metabolites, and when both are equivalent in efficacy and safety, the inhibition or induction has little therapeutic implications [21].

Besides coadministered drugs, also the patient's physiologic status (age, gender, and comorbidity) and his/her genetic polymorphisms may be responsible for different drug-metabolizing enzyme activity [21]. It is estimated that genetics may explain $20-95 \%$ of variability in the therapeutic response and toxicity $[21,22]$.

\section{The Particular Case of TMX}

Among the ANs, TMX is the one more extensively studied for drug interactions with ADs. TMX is a selective modulator of estrogen receptors [12]. In women with breast cancer and at the initial stage, the use of TMX has been associated with a reduced risk of recurrence at about
$1 / 2$ and death risk by breast cancer at about $1 / 3$ [12]. TMX is converted to endoxifen, its ActiveMet, by the most polymorphic and well-studied gene of the CYP 450 system: CYP 2D6 [12]. More than 80 different major alleles were identified, many of which are responsible for increased or reduced activity of CYP 2D6 [15, 23-26]. Thus, based on the genotype, individuals can be divided into weak, intermediate, extensive or very extensive metabolizers [15]. The CYP 2D6 lack of activity compromises TMX efficacy by reducing its bioactivation to endoxifen $[15,23-26]$.

Several studies have shown that drugs that inhibit CYP 2D6 can reduce the clinical benefit of TMX $[27,28]$. SSRIs inhibit, to varying degrees, CYP 2D6 [19] (table 1). Pharmacological inhibition of CYP 2D6 has also been associated with worse breast cancer prognosis. This observation is consistent with the critical role of CYP 2D6 in the metabolic activation of TMX and puts in evidence a drug interaction that can and should be avoided.

\section{Interactions between ADs and Cytostatics}

Drug interactions can result in clinically significant changes in pharmacokinetics and/or pharmacodynamics of ANs altering their therapeutic efficacy and toxicity. Concerning ANs, at present, the majority of information available comes from animal experiments or in vitro tests [21]. Despite these limitations, research has provided valuable information on the pharmacokinetics of either ADs or ANs, allowing the integration of these data to predict possible drug interactions between these two categories of drugs.

Recent studies focusing on the inhibitory properties of tricyclic ADs are scarce. However, there is some evidence on their moderate to high inhibitory potential on CYP 2C19 and CYP 2D6 isoenzymes [29, 30]. Much more information is available about SSRIs. Besides markedly inhibiting CYP 2D6, fluoxetine inhibits moderately CYP 2C19 and 2C9 isoenzymes [14, 19, 31]. Sertraline can also cause moderate inhibition of CYP 2C19 [32] and fluvoxamine is a strong inhibitor of CYP 1A2, 2C9 and 2C19 isoenzymes $[14,31]$. As for paroxetine, its inhibitory potential is high for both CYP 2D6 and 2B6 [14, 31]. All these drugs also inhibit, to a mild degree, CYP $3 \mathrm{~A} 4$ isoenzyme $[14,19,31,32]$.

Citalopram, escitalopram, venlafaxine and mirtazapine are among the ADs whose inhibitory potential is smaller [14, 31]. Milnacipran stands out for minimal he- 
Table 1. Choice among ADs in patients with a history of breast cancer on therapy with TMX

\begin{tabular}{lll}
\hline $\begin{array}{l}\text { Antidepressant } \\
\begin{array}{l}\text { Venlafaxine } \\
\text { Mirtazapine }\end{array}\end{array}$ & $\begin{array}{l}\text { Inhibition } \\
\text { of CYP 2D6 }\end{array}$ & Advice based on evidence \\
\hline Citalopram, escitalopram & slight & $\begin{array}{l}\text { The safest choice with TMX } \\
\text { Direct studies are lacking with TMX }\end{array}$ \\
\hline $\begin{array}{l}\text { Duloxetine, sertraline, } \\
\text { fluvoxamine }\end{array}$ & moderate & $\begin{array}{l}\text { Only citalopram or sertraline were studied directly with TMX, so the risk of decreasing } \\
\text { endoxifen must be weighed against the benefits of these ADs }\end{array}$ \\
$\begin{array}{l}\text { Paroxetine, fluoxetine, } \\
\text { buproprion }\end{array}$ & strong & Avoid to combine with TMX \\
\hline
\end{tabular}

Adapted from Desmarais and Looper [19].

patic metabolization (limited to conjugation with glucuronic acid) being its involvement in pharmacokinetic intermediate metabolizers [33-35] unlikely.

Regarding the potential pharmacokinetic drug interactions between ANs and ADs and particularly regarding the risk of toxicity and loss of effectiveness of ANs, the combined analysis of the AD pharmacokinetic data and the known evidences about AN metabolization (table 2) support the following considerations.

(1) Altretamine and lomustine are subject to extensive hepatic metabolization with the generation of ActiveMet [36]. The isoenzymes of CYP 450 involved in the biotransformation of these ANs are still unknown [36]. However, with fluoxetine, fluvoxamine and paroxetine, moderate to powerful inhibitors of multiple isoenzymes of CYP 450 $[14,31]$, the reduced effectiveness of these ANs, when coadministered with these ADs, is foreseeable.

(2) The evidence based on animal studies does not suggest that busulfan and chlorambucil are metabolized by the system CYP 450 [36]. Regarding estramustine, mechlorethamine, melphalan, temozolomide, 5-fluorouracil, gemcitabine, mercaptopurine, thioguanine, cisplatin, carboplatin, oxaliplatin, daunorubicin, doxorubicin, epirubicin and vorinostat, to date, there is no evidence of the involvement of the system CYP 450 in the metabolization of these ANs, suggesting that in these cases drug interaction with ADs is rare [36].

(3) Cyclophosphamide and procarbazine are metabolized by CYP 2B6 to ActiveMets [36]. The concomitant use of an inhibitor of CYP 2B6 (e.g. paroxetine to a greater degree and fluoxetine, sertraline, fluvoxamine and buproprion to a lesser degree $[14,31])$ can reduce the effectiveness of these ANs. In the particular case of cyclophos- phamide, which is subject to metabolization by CYP 2C19 generating its ActiveMet, the CYP 2C19 inhibitors (e.g. sertraline [32], fluoxetine [14, 31], fluvoxamine [14, 31] and tricyclic ADs [35]) can also reduce its effectiveness.

On the other hand, the biotransformation of cyclophosphamide via CYP 3A4 leads to the formation of inactive metabolites. Therefore, by the use of CYP 3A4 inhibitors more active drug may be available (increased efficacy/toxicity ratio) and the coadministration with a CYP 3A4 inducer can be translated into higher neurotoxicity [36]. Procarbazine is subject, to a lesser extent, to metabolization in CYP 1A [36] with the formation of ActiveMet; therefore, using this isoenzyme's inhibitors (e.g. fluvoxamine [14]) can also reduce the effectiveness of this AN [29]. The same applies to dacarbazine whose route of biotransformation matches that of procarbazine [36].

(4) Ifosfamide, thiotepa, toremifene and sunitinib are metabolized by CYP 3A4 in ActiveMet [36]. The inhibitors of this isoenzyme (i.e. fluoxetine, sertraline, paroxetine and fluvoxamine, even with slight inhibitory potential [14]) may impair the activation of the ANs compromising its therapeutic effect.

Docetaxel and paclitaxel are also metabolized by CYP 3A4. For these ANs, the use of a CYP 3A4 inhibitor may increase the plasma concentration of taxanes causing increased toxicity (e.g. myelosuppression, constitutional symptoms and peripheral neuropathy) [36]. The same occurs with the other ANs in the antimicrotubules category (vinblastine, vincristine, vindesine, vinorelbine), corticosteroids, etoposide, irinotecan and sorafenib whose overlap use with CYP 3A4 inhibitors can increase toxicity, and with CYP 3A4 inducers reduce efficacy [36]. In the case 
Table 2. Metabolism of ANs categorized by major pharmacological classes

\begin{tabular}{|c|c|c|}
\hline Antineoplastic agents & Metabolism & Probable drug interaction with INDs or INBs \\
\hline \multicolumn{3}{|l|}{ Alkylating agents } \\
\hline Altretamine & Hepatic oxidation $\rightarrow$ (ActiveMet) & \\
\hline \multicolumn{3}{|l|}{ Busulfan } \\
\hline \multicolumn{3}{|l|}{ Chlorambucil } \\
\hline \multirow[t]{2}{*}{ Cyclophosphamide } & CYP 2B6 > 2C19 & IND 2 B6 may $\uparrow$ ActiveMet; INB 2B6 $\downarrow$ D efficacy; IND 3A4 can $\uparrow$ \\
\hline & (ActiveMet); CYP3A4 (ActiveMet) & neurotoxicity, INB 3A4 may provide more active D ( $\uparrow$ Efficacy/toxicity) \\
\hline \multirow[t]{2}{*}{ Dacarbazine } & CYP $1 \mathrm{~A} 2>2 \mathrm{E} 1$ & INB CYP $1 \mathrm{~A} 2$ and $2 \mathrm{E} 1$ may $\downarrow$ ActiveMet \\
\hline & (ActiveMet is a methylated DNA) & \\
\hline Estramustine & $\mathrm{O}$ & \\
\hline Ifosfamide & & $\begin{array}{l}\text { CYP3A4 IND may } \uparrow \mathrm{D} \text { activation and generated more metabolites, with } \\
\text { neurotoxic potential; CYP } 3 \mathrm{~A} 4 \text { INB } \downarrow \mathrm{D} \text { activation and its efficacy }\end{array}$ \\
\hline Lomustine & Extensive hepatic oxidation $\rightarrow$ (ActiveMet) & Potential interaction with CYP 450 INB ( $\uparrow$ pro-D, $\downarrow$ efficacy and $\uparrow$ toxicity) \\
\hline Mechlorethamine & O & \\
\hline Melphalan & ○ & \\
\hline Procarbazine & CYP 2B6 > 1 A (ActiveMet) & CYP 2 B6 or CYP 1A INB may $\downarrow$ D efficacy \\
\hline Thiotepa & CYP 3A4 > 2B6 (ActiveMet) & 3A4 IND may $\uparrow$ ActiveMet, INB may $\downarrow$ D efficacy \\
\hline Temozolomide & $\mathrm{O}$ & \\
\hline \multicolumn{3}{|l|}{ Antimetabolites } \\
\hline \multirow[t]{2}{*}{ Capecitabine } & 5-Fluorouracil Pro-D & Potential to $\uparrow \mathrm{CYP} 2 \mathrm{C} 9$ substrate concentrations \\
\hline & CYP 2C9 INB potential & \\
\hline Fludarabine & $\odot$ & \\
\hline 5-Fluorouracil & O & \\
\hline Gemcitabine & O & \\
\hline Mercaptopurine & O & \\
\hline Methotrexate & Hepatic metabolism $\rightarrow$ & \\
\hline Thioguanine & O & \\
\hline \multicolumn{3}{|l|}{ Antimicrotubules } \\
\hline Docetaxel & CYP $3 \mathrm{~A} 4$ & $\begin{array}{l}\text { CYP 3A4 INB may } \uparrow \text { taxanes (myelosuppression and peripheral neutropenia); } \\
\text { CYP 3A4 IND may } \downarrow \text { taxanes; }\end{array}$ \\
\hline Paclitaxel & CYP 2C8 > CYP3A4 & CYP3A4 INB may $\uparrow$ paclitaxel (myelosuppression and peripheral neutropenia) \\
\hline Vinblastine & CYP 3A4 & Probable $\uparrow$ with CYP 3A4 INB \\
\hline $\begin{array}{l}\text { Vincristine, vindesine, } \\
\text { vinorelbine }\end{array}$ & CYP 2D6 INB potential & (myelosuppression and peripheral neutropenia) and $\downarrow$ with IND \\
\hline \multicolumn{3}{|l|}{ Corticosteroids } \\
\hline Dexamethasone & CYP 3A4 (InactiveMet) & Toxicity risk with CYP 3 A 4 INB and $\downarrow$ efficacy with CYP 3 A4 IND \\
\hline $\begin{array}{l}\text { Methylprednisolone, } \\
\text { prednisolone, prednisone }\end{array}$ & Dexamethasone is a CYP $3 \mathrm{~A} 4 \mathrm{IND}$ & \\
\hline \multicolumn{3}{|c|}{ Hormone agonists/antagonists } \\
\hline \multirow[t]{2}{*}{ Anastrazole } & $\rightarrow(\mathrm{CYP} 3 \mathrm{~A} 4$ is possible $)$ & \\
\hline & CYP $1 \mathrm{~A} 2,2 \mathrm{C} 8,2 \mathrm{C} 9$ and $3 \mathrm{~A} 4 \mathrm{INB}$ potential & \\
\hline Exemestane & CYP 3A4 (InactiveMet) & May $\uparrow$ with CYP 3 A4 INB; CYP3A4 IND may $\downarrow$ efficacy \\
\hline \multirow[t]{2}{*}{ Letrozole } & CYP 2 A6 e CYP 3A4 (MetInactivo); & May $\uparrow$ with CYP 3 A4 INB; CYP3A4 IND may $\downarrow$ efficacy \\
\hline & CYP 2 A6 and 2C19 INB & \\
\hline Tamoxifen & CYP 2D6 (ActiveMet) CYP 3A4 INB & $\begin{array}{l}\text { CYP2D6 INB may } \downarrow \text { efficacy; CYP } 3 \text { A4 IND may } \uparrow \text { risk of side effects } \\
\text { (e.g. hot flushes, nausea, vomiting) }\end{array}$ \\
\hline Toremifene & CYP 3A4 (ActiveMet) & CYP 3A4 INB may $\downarrow$ efficacy \\
\hline \multicolumn{3}{|l|}{ Platinum compounds } \\
\hline $\begin{array}{l}\text { Cisplatin, carboplatin, } \\
\text { oxaliplatin }\end{array}$ & O & \\
\hline \multicolumn{3}{|l|}{ Topoisomerase inhibitors } \\
\hline Daunorubicin & 0 & \\
\hline Doxorubicin & (CYP $3 \mathrm{~A} 4$ is possible) & \\
\hline Epirubicin & $\bigcirc$ & \\
\hline Etoposide & CYP $3 \mathrm{~A} 4>$ CYP 2E1, $1 \mathrm{~A} 2$ & May $\uparrow$ with CYP $3 \mathrm{~A} 4$ INB $(\uparrow \mathbf{x}$ myelosuppression, mucositis) \\
\hline Idarubicin & $\bigcirc$ & \\
\hline Irinotecan & CYP 3A4 (ActiveMet) & 3A4 INB may $\uparrow$ formation of SN-38 ( $\uparrow \mathbf{x}$ myelosuppression $)$ \\
\hline & & 3A4 IND may $\uparrow$ conversion of SN-38 to InactiveMet ( $\downarrow$ Efficacy) \\
\hline Mitoxantrone & $\rightarrow$ & \\
\hline Topotecano & $\rightarrow$ & \\
\hline
\end{tabular}


Table 2 (continued)

\begin{tabular}{|c|c|c|}
\hline Antineoplastic agents & Metabolism & Probable drug interaction with INDs or INBs \\
\hline \multicolumn{3}{|c|}{ Tyrosine kinase inhibitors and monoclonal antibodies } \\
\hline Bortezomib & CYP 3A4, 2C19, 1A2, 2D6 and 2C9 & Potential for $\uparrow$ or $\downarrow$ with CYP $3 \mathrm{~A} 4$ and $2 \mathrm{C} 19$ strong INB or IND \\
\hline Dasatinib & $\begin{array}{l}\text { CYP 3A4 } \\
\text { (ActiveMet, with activity similar to dasatinib) }\end{array}$ & $\uparrow$ dasatinib with CYP 3 A4 INB $(\uparrow \mathbf{x})$ \\
\hline Erlotinib, gefitinib & CYP 3A4 & May $\uparrow$ with CYP 3A4 INB \\
\hline Imatinib & CYP $3 \mathrm{~A} 4$ & $\begin{array}{l}\text { x When conjugated with CYP } 3 \mathrm{~A} 4 \mathrm{INB} \text { (fluid retention, weight gain, nausea, } \\
\text { vomiting, neutropenia) }\end{array}$ \\
\hline Sorafenib & CYP $3 \mathrm{~A} 4$ & May $\downarrow$ efficacy with CYP 3 A4 IND \\
\hline Sunitinib & CYP 3A4 (ActiveMet) & May $\uparrow$ with CYP 3 A4 INB and $\downarrow$ efficacy by the ActiveMet $\downarrow$ \\
\hline
\end{tabular}

Other anticancer drugs

Cytotoxic antibiotics:

bleomycin, dactinomycin,

mitomycin

Histone deacetylase

inhibitor: vorinostat

Mammalian target of

rapamycin (mTOR)

INB CYP potential suggested

○

CYP 3A4 (5 ActiveMet)

inhibitor: temsirolimus

InactiveMet $=$ Inactive metabolite; $\uparrow=$ increase; $\downarrow$ = decrease; $\rightarrow=$ exact CYP450 isoenzyme unknown; $\mathbf{\square}=$ animal data suggest no involvement of CYP 450; $\bigcirc=$ involvement of CYP 450 in the metabolism is unlikely; $\odot=$ quickly converted into ActiveMet (drug interaction potential mediated by CYP appears to be minimal); $\mathbf{x}=$ toxicity; INB = inhibitor; IND = inductor; $\mathrm{D}=$ antineoplastic drug; > = metabolization more extensive than. Adapted from Scripture and Figg [21] and Tony and Tseng [36].

of exemestane and letrozole, the likely toxicity with the use of CYP 3A4 inhibitors is translated into myalgia, constitutional symptoms, peripheral edema and hot flushes [29]. Under these conditions, regarding imatinib, the most prominent adverse symptoms are the weight gain, nausea, vomiting and neutropenia [36]. The toxicity of dasatinib can also increase with the coadministration of CYP 3A4 inhibitors [36].

\section{Conclusion}

In oncological patients, the $\mathrm{AD}$ choice is a swing where the profile of side effects and pharmacokinetics should be well weighed. Drug interactions between ADs and ANs can compromise the effectiveness of ANs and increase their toxicity with obvious prognostic implications. The information available about the metabolization of $\mathrm{ADs}$ and ANs with emphasis on the CYP 450 system facilitates drug interaction prediction, which could be avoided if undesirable.

The pharmacokinetic drug interactions with ADs are unlikely with busulfan, chlorambucil, estramustine, mechlorethamine, melphalan, temozolomide, 5-fluorouracil, gemcitabine, mercaptopurine, thioguanine, cisplatin, carboplatin, oxaliplatin, daunorubicin, doxorubi- cin, epirubicin and vorinostat. Among the remaining ANs considered here, the risk of loss of efficacy or increased toxicity, when coadministered with certain ADs, is a possibility. Venlafaxine, mirtazapine, citalopram and escitalopram are small inhibitors of CYP 2D6, therefore being a safe choice when using TMX. Most of the other ANs subjected to metabolization by CYP $4503 \mathrm{~A} 4$ should be used with caution concomitantly with inhibitors of this isoenzyme such as fluoxetine, sertraline, paroxetine and fluvoxamine. Escitalopram, citalopram, venlafaxine, mirtazapine and milnacipram are ADs with minimal CYP 450 inhibitory potential and are therefore safer in these patients.

\section{Limitations of This Review}

Regarding drug interactions between ANs and ADs, neither pharmacodynamic phenomena nor hepatic conjugation reactions have been considered. The results proposed here are from the integration of evidence known about the individual pharmacokinetics of the ADs and ANs. These are, however, assumptions that need additional support. In fact, with the exception of extended research on TMX, the literature lacks evidence on the impact of different ADs in plasma concentrations of ANs (and its metabolites) and its anticancer efficacy and toxicity. 


\section{References}

1 Pasquini M, Biondi M, Costantini A, Cairoli F, Ferrarese G, Picardi A, Sternberg C: Detection and treatment of depressive and anxiety disorders among cancer patients: feasibility and preliminar findings from a Liason Service in an Oncology division. Depress Anxiety 2006;23:441-448.

2 Grassi L, Nanni MG, Uchitomi Y, Riba M, Kissane DW, Maj M, Sartorius N: Pharmacotherapy of depression in people with cancer; in Kissane DW, Maj M, Sartorius N (eds): Depression and Cancer. Chichester, Wiley, 2010

3 Carvalho VA, Macieira RC, Liberato RP, Veit MT, Kovács MJ, Gomes MJB, et al: Temas em psico-oncologia. São Paulo, Summus Editorial, 2008

4 Massie MJ: Prevalence of depression in patients with cancer. J Natl Cancer Inst Monogr 2004;32:57-71.

5 Grassi L, Holland JC, Johansen C, Koch U, Fawzy F: Psychiatric concomitants of cancer, screening procedures, and training of health care professionals in oncology: the paradigms of psycho-oncology in the psychiatry field; in Christodoulou GN (ed): Advances in Psychiatry. Athens, Beta Publishing Athens, 2005.

6 Hjerl K, Andersen EW, Keiding N, Mouridsen HT, Mortensen PB, Jorgensen T: Depression as a prognostic factor for breast cancer mortality. Psychosomatics 2003;44:24-33.

$\checkmark 7$ Stommel M, Given BA, Given CW: Depression and functional status as predictors of death among cancer patients. Cancer 2002; 94:2719-2727.

$\checkmark 8$ Okamura M, Akizuki N, Nakano T, Shimizu $\mathrm{K}$, Ito T, Akechi T, Uchitomi Y: Clinical experience of the use of a pharmacological treatment algorithm for major depressive disorder in patients with advanced cancer. Psychooncology 2008;17:154-160.

9 Rayner L, Rayner L, Price A, Hotopf M, Higginson IJ: The management of depression in palliative care. Eur J Cancer Care 2011;47: 702-712.

-10 Stiefel FC, Komblith AB, Holland JC: Changes in prescription patterns of psychotropic drugs for cancer patients during a 10-year period. Cancer 1990;65:1048-1053.

-11 Rodin G, Katz M, Lloyd N, Green E, Mackay JA, Wong RK: Treatment of depression in cancer patients. Curr Oncol 2007;14:180188.

-12 Kalash GR: Psychotropic drug metabolism in cancer patients: clinical aspects of management of potential drug interactions. Psychooncology 1998;7:307-320.
13 Yap KY, Ho YX, Chui WK, Chan A: Harnessing the internet cloud for managing drug interactions with chemotherapy regimens in patients with cancer suffering from depression. Acta Oncol 2010;49:1235-1245.

14 Ciraulo DA, Shader RI, Greenblatt DJ, Creelman WL: Drug Interactions in Psychiatry, ed 3. Philadelphia, Lippincott Williams \& Wilkins, 2006.

15 Tatro DS: Drug Interactions Facts: The Authority on Drug Interactions. St Louis, Facts \& Comparations, 2008

16 Richelson E: Pharmacokinetic drug interactions of new antidepressants: a review of the effects on metabolism of other drugs. Mayo Clin Proc 1997;72:835-847.

17 Haddad A, Davis M, Lagman R: The pharmacological importance of cytochrome CYP3A4 in palliation of symptoms: review and recommendations for avoiding adverse drug interactions. Support Care Cancer 2007;15:251-257.

18 Kelly MC, Juurlink DN, Gomes T, DuonHua M, Pritchard KI, Austin PC, Paszat LF Selective serotonin reuptake inhibitors and breast cancer mortality in women receiving tamoxifen: a population cohort study. BMJ 2010;340:c693

19 Desmarais JE, Looper KJ: Interactions between tamoxifen and antidepressants via cytochrome P450 2D6. J Clin Psychiatry 2009; 70:1688-1697.

20 Barreiro RJ, Siva JFM, Fraga CA: Noções básicas do metabolismo de fármacos. Rev Química Nova 1996;19:441-451.

21 Scripture DC, Figg WD: Drug Interactions in Cancer Therapy: Pharmacokinetic Interactions: Metabolism. 2006. http:// www.medscape.com/viewarticle/540883_4 (accessed May 9, 2011).

22 Evans WE, McLeod HL: Pharmacogenomics - Drug disposition, drug targets, and side effects. N Engl J Med 2003;348:538-549.

23 Schroth W, Antoniadou L, Fritz P, Schwab M, Muerdter T, Zanger UM, et al: Breast cancer treatment outcome with adjuvant tamoxifen relative to patient CYP2D6 and CYP2C19 genotypes. J Clin Oncol 2007; 25:5187-5193.

-24 Schroth W, Goetz MP, Hamann U, Fasching PA, Schmidt M, Winter S, et al: Association between CYP2D6 polymorphisms and outcomes among women with early stage breast cancer treated with tamoxifen. JAMA 2009; 302:1429-1436.

25 Kiyotani K, Mushiroda T, Sasa M, Bando Y, Sumitomo I, Hosono N, et al: Impact of CYP2D6* 10 on recurrence-free survival in breast cancer patients receiving adjuvant tamoxifen therapy. Cancer Sci 2008;99:995999.
26 Xun Y, Sun Y, Yao L, Shi L, Wu Y, Ouyang T, et al: Association between CYP2D6 ${ }^{*} 10$ genotype and survival of breast cancer patients receiving tamoxifen treatment. Ann Oncol 2008;19:1423-1429.

27 Jin Y, Desta Z, Stearns V, Ward B, Ho H, Lee $\mathrm{KH}$, et al: CYP2D6 genotype, antidepressant use, and tamoxifen metabolism during adjuvant breast cancer treatment. J Natl Cancer Inst 2005;97:30-39.

-28 Borges S, Desta Z, Li L, Skaar TC, Ward BA, Nguyen A, et al: Quantitative effect of CYP2D6 genotype and inhibitors on tamoxifen metabolism: implication for optimization of breast cancer treatment. Clin Pharmacol Ther 2006;80:61-74

29 Yue QY, Säwe J: Different effects of inhibitors on the $\mathrm{O}$ - and $\mathrm{N}$-demethylation of codeine in human liver microsomes. Eur J Clin Pharmacol 1997;52:41-47.

30 Shin JG, Parks JY, Kim MJ, Shon JH, Yoo YR, Cha IJ, et al: Inhibitory effects of tricyclic antidepressants (TCAs) on human cytochrome p450 enzymes in vitro: mechanism of drug interaction between TCAs and phenytoin. Drug Metab Dispos 2002;30:1102-1107.

31 Flockhart DA: Drug Interactions: Cytochrome P450 Drug Interaction Table. Indiana University School of Medicine. 2007. http://medicine.iupui.edu/clinpharm/ddis/ table.asp (accessed May 9, 2011).

32 Obach RS, Cox LM, Tremaine LM: Sertraline is metabolized by multiple cytochrome P450 enzymes monoamine oxidases, and glucuronyl transferases in human: an in vitro study. Drug metabolism and disposition. Drug Metab Dispos 2005;33:262-270.

33 Kazuhisa T, Kazuyuki T, Katsuya O, Satoko K, Noriaki S, Yasufumi S: Examination of drug-drug interaction of milnacipran hydrochloride in the presence of human P-450. Iyakuhin Kenkyu 2000;31:659-667.

>34 Puozzo C, Lens S, Reh C, Michaelis K, Rosillon D, Deroubais X, et al: Lack of interaction of milnacipran with the cytochrome P450 isoenzymes frequently involved in the metabolism of antidepressants. Clin Pharmacokinet 2005;44:977-988.

35 Savella (Milnacipran Hydrochloride) - Drug Interactions, Contraindications, Overdosage. 2009. http://www.druglib.com/druginfo/ savella/interactions_overdosage_contraindications (accessed May 9, 2011).

36 Tony A, Tseng A: Potential Interactions between Antineoplastics and Antiretrovirals. 2011. www.hivclinic.ca/main/drugs_ interact_files/Chemo-int.pdf (accessed May 9, 2011) 